\section{Neues kombiniertes Verfahren zur Behandlung atropher Narben}

\begin{abstract}
Die Subzision (subcutaneous incisionless surgery), also die Durchtrennung verkürzter Bindegewebsstränge mit feinen Kanülen oder Drähten, wird zur Behandlung von Falten und eingezogenen Narben eingesetzt. Die Methode hat sich einen festen Platz in der ästhetischen Dermatologie erobert. Allerdings senkt sich das Gewebe häufig erneut ab, weshalb oft zusätzlich unterfüttert werden muss. Eine gezielte Vakuumtherapie könnte weiterhelfen.
\end{abstract}

$\mathrm{D}$ ermatologen der Universitätsklinik in Teheran überlegten, ob sich das Einziehen der Haut nach der Intervention verhindern ließe, indem man die behandelten Stellen mit der Saugpumpe eines Geräts zur Mikrodermabrasion behandelt. Die Hypothese: wiederholtes Einwirken des Vakuums auf die Narben im Anschluss an die Subzision könnte immer neue Hämatome in der geschaffenen Tasche induzieren, den Abheilungsprozess verzögern und für die Bildung von mehr neuem Bindegewebe sorgen.

Sie prüften diese Möglichkeit bei 58 Patienten mit atrophen Akne- oder
Windpockennarben, Narben nach Traumata oder eingezogenen Operationsnarben. Der minimal invasive Eingriff wurde dazu ergänzt durch serielle SaugProzeduren, die ab dem dritten Tag nach der Subzision zwei Wochen lang täglich oder jeden zweiten Tag durchgeführt wurden.

Eine effektive Anwendung des Vakuums (pro Einzelanwendung jeweils für höchstens 4 Sekunden, pro Sitzung bis zu 12 Applikationen) induzierte Ödeme und Hämatome, was den Narbengrund für kurze Zeit über das Niveau der umgebenden Haut anhob.
Eine signifikante Verbesserung um $70 \%$ (bezogen auf Tiefe und Größe der Narbe) erzielte man in der Subgruppe mit 46 Patienten, die das volle Therapieprogramm absolvierten. Ein gutes Viertel dieser Patienten hatte sogar exzellente Therapieerfolge von über $80 \%$ iger Narbenverkleinerung. Bei spätem Beginn der Ansaug-Prozeduren oder langen Intervallen zwischen den Sitzungen war die kombinierte Behandlung nicht so erfolgreich (im Mittel: 43\%ige Verbesserung).

Fazit: Mehrfach wiederholte kurzzeitige Anwendungen von Unterdruck nach einer Subzisionsbehandlung atropher Narben im Gesicht scheinen die Effektivität des Eingriffs zu erhöhen.

Waltraud Paukstadt

Aalami Harandi S et al. Subcision-suction method: a new successful combination therapy in treatment of atrophic acne scars and other depressed scars. J Eur Acad Dermatol Venereol 2011; 25: 92-9

\title{
Blick über die Grenzen: Wenn die Schwester das Rezept ausstellt ...
}

Früher hieß sie Gemeindeschwester. Heute ist sie als Fachkraft für integrierte Versorgung, als Versorgungsassistentin in der Hausarztpraxis, Fallmanagerin oder mobile Praxisassistentin wieder im Kommen. Die Effizienz der Betreuung von Hautpatienten durch nicht-ärztliches Personal mit spezieller dermatologischer Qualifikation untersuchten britische Autoren.

n den Niederlanden, Skandinavien und im angelsächsischen Raum haben Pflegekräfte eine andere Stellung im Gesundheitssystem. Die Übernahme ärztlicher Aufgaben durch sie ist dort nicht nur zur Sicherstellung der medizinischen Versorgung in ländlichen Gebieten Usus.

In Großbritannien bildet man auch spezialisierte Schwestern und Pfleger für die Krankenversorgung in der Rheumatologie sowie in der Dermatologie aus. In den letzten Jahren wurden dort besonders ausgebildeten Pflegekräften immer mehr Versorgungsaufgaben übertragen, und mittlerweile gibt es auf der Insel über 19.000 Schwestern mit vollem Verschreibungsrecht.
Unabhängig von der oft erheblichen Beeinträchtigung durch eine Dermatose ist mangelnde Compliance ein großes Problem bei der Therapie chronischer Hautkrankheiten. Vor diesem Hintergrund untersuchten britische Kliniker, wie sich die Behandlung durch die speziell ausgebildeten Krankenschwestern mit Verschreibungskompetenz aus Sicht der Patienten auswirkt. 42 konsekutiv behandelte Patienten mit Akne, Psoriasis oder Ekzem, wurden befragt.

Die Patienten waren überzeugt, dass das „nurse prescribing“ sowohl den $\mathrm{Zu}$ gang zu guter dermatologischer Versorgung verbessert als auch deren Effizienz erhöht. Großen Wert legten die Befragten darauf, „ihre“ Schwester telefonisch erreichen zu können, und auf die Wohnortnähe. Informationsaustausch und Beteiligung an Therapieentscheidungen sorgten für gutes Verständnis des Therapieplans und stärkten die Compliance.

Die praktische Erfahrung der Schwester, ein interaktiver und engagierter Konsultationsstil sowie die Möglichkeit, ein persönliches Verhältnis zu ihr aufzubauen, erhöhten die Zufriedenheit mit der Behandlung und die Mitarbeit der Patienten.

Fazit: Bei den Briten verbessert die Regelung, die es besonders qualifizierten Schwestern erlaubt, Rezepte auszustellen, den Zugang zu dermatologischer Versorgung und die Versorgungsqualität.

Waltraud Paukstadt

Courtenay $\mathrm{M}$ et al. Patients' views of nurse prescribing: effects on care, concordance and medicine taking. Br J Dermatol. 2011; 164: 396-401 